\title{
Genetic and Molecular Analyses in Crosses of Race 2 and Race 7 of Albugo candida
}

\author{
Tika B. Adhikari, Jean Q. Liu, Snehlata Mathur, Chunren X. Wu, and S. Roger Rimmer
}

First author: Crop Production and Pest Control Research, U.S. Department of Agriculture-Agricultural Research Service, Department of Botany and Plant Pathology, Purdue University, 915 W. State Street, West Lafayette, IN 47907; second author: Pioneer Hi-Bred International, Inc., Research and Product Development, 7300 N.W. 62nd Ave., Johnston, IA 50131; third author: Pacific Agri-Food Research Centre, Agriculture and Agri-Food Canada, P.O. Box 1000, Agassiz, BC, V0M 1A0, Canada; fourth author: Monsanto Canada Inc., P.O. Box 250, 1885A Mitchell Road South, Listowel, Ontario, N4W 3H2, Canada; and fifth author: Saskatoon Research Centre, Agriculture and Agri-Food Canada, 107 Science Place, Saskatoon, SK, S7N 0X2, Canada.

Accepted for publication 11 March 2003.

\section{ABSTRACT}

Adhikari, T. B., Liu, J. Q., Mathur, S., Wu, C. X., and Rimmer, S. R. 2003. Genetic and molecular analyses in crosses of race 2 and race 7 of Albugo candida. Phytopathology 93:959-965.

The inheritance of avirulence and polymorphic molecular markers in Albugo candida, the cause of white rust of crucifers, was studied in crosses of race 2 (Ac2), using isolates MiAc2-B1 or MiAc2-B5 (metalaxylinsensitive and virulent to Brassica juncea cv. Burgonde) with race 7 (Ac7), using isolate MsAc7-A1 (metalaxyl-sensitive and virulent to $B$. rapa cv. Torch). Hybrids were obtained via co-inoculation onto a common susceptible host. Putative $F_{1}$ progeny were selfed to produce $F_{2}$ progeny. The parents and $F_{1}$ progeny were examined for virulence on the differential cultivars $B$. juncea cv. Burgonde and B. rapa cv. Torch. Segregation of avirulence or virulence of $\mathrm{F}_{2}$ populations was analyzed on $\mathrm{cv}$.
Torch. Putative $F_{1}$ hybrids were confirmed by random amplified polymorphic DNA markers specific for each parent. Avirulence or virulence of $\mathrm{F}_{2}$ progeny to $B$. rapa $\mathrm{cv}$. Torch suggested $3: 1$ in each of three populations, supporting the hypothesis of a single dominant avirulence gene. Amplified fragment length polymorphism markers also segregated in regular Mendelian fashion among $\mathrm{F}_{2}$ progeny derived from two $\mathrm{F}_{1}$ hybrids ( $\mathrm{Cr} 2-5$ and $\mathrm{Cr} 2-7)$ of Cross-2. This first putative avirulence gene in A. candida was designated AvrAcl. These results suggest that a single dominant gene controls avirulence in race Ac2 to B. rapa cv. Torch and provides further evidence for the gene-for-gene relationship in the Albugo-Brassica pathosystem.

Additional keyword: specificity.
Albugo candida (Pers.) Kunze, a diploid, oomycete biotroph, causes white rust of cultivated Brassica spp. At least 10 pathogenic races (pathotypes) of $A$. candida have been identified on the basis of specificity to different species of crucifers $(5,13,27,28)$. Among the races, race 2 (hereafter Ac2) causes severe symptoms on most cultivars of brown mustard (Brassica juncea [L.] Czern. and Coss.), but also infects some genotypes of other Brassica spp., including oilseed turnip rape, Brassica rapa L. (25-27). Race 7 (hereafter Ac7) is largely restricted to $B$. rapa, but has been reported to cause disease on some cultivars of B. napus (7), and some genotypes of $B$. juncea (25).

The genetic control of resistance to $A$. candida has been studied in several Brassica spp. For example, dominant alleles at three unlinked loci $\left(A c 7_{1}, A c 7_{2}\right.$, and $\left.A c 7_{3}\right)$ conferred resistance in $B$. napus cv. Regent to race Ac7 of $A$. candida $(7,19)$. Two loci also controlled resistance in B. napus to A. candida race Ac2 collected from B. juncea (32). The Chinese B. napus accession 2282-9, which is susceptible to Ac7, has one locus controlling resistance to an isolate of $A$. candida collected from $B$. carinata (20). These studies indicated that only one allele for resistance was sufficient to condition an incompatible reaction in this pathosystem (8). In addition, a single locus controlling resistance to Ac2 in B. napus and $B$. rapa was mapped using restriction fragment length poly-

Corresponding author: S. R. Rimmer; E-mail address: RimmerR@agr.gc.ca

Publication no. P-2003-0527-01R

This article is in the public domain and not copyrightable. It may be freely reprinted with customary crediting of the source. The American Phytopathological Society, 2003 morphism (RFLP) markers $(8,16)$. Recently, a dominant allele at a single locus or two tightly linked loci was reported to confer resistance to both races Ac2 and Ac7 of A. candida (17).

In contrast to our knowledge of the genetics of resistance to $A$. candida in Brassica spp., information on the genetics of the pathogen is minimal and genetic studies were considered intractable due to the lack of a reliable method to germinate oospores. Liu and Rimmer (21) developed an efficient method to germinate oospores of $A$. candida and isolated metalaxyl-insensitive strains derived from Ac2 and Ac7. These developments provided an opportunity to analyze genetically the inheritance of cultivar specificity and metalaxyl resistance and to conduct other genetic studies of $A$. candida. Among oomycete pathosystems, most studies have been focused on heterothallic species such as Phytophthora infestans (29), P. parasitica (10), and Bremia lactucae (3,14, $22,24)$. For homothallic oomycetes, where inbreeding is common, occasional outcrossing has been reported $(11,34)$ and used to produce $\mathrm{F}_{2}$ progeny in which both avirulence genes and molecular markers segregated in a Mendelian fashion $(31,35)$.

The use of molecular markers based on the polymerase chain reaction (PCR), such as random amplified polymorphic DNA (RAPD) (36), is advantageous because it requires no prior information about genomic sequences and is simple compared with other molecular marker methods. RAPD markers are usually dominant because polymorphisms are detected as the presence or absence of bands. RAPDs were used successfully as genetic markers in genetic studies of oomycetes $(11,31,35)$. Amplified fragment length polymorphism (AFLP) markers also have been used for genetic analysis and genome mapping of both plants and pathogens (33). AFLP markers are allele specific and the technique allows the simultaneous analysis of a large number of DNA 
bands per gel. No previous study has examined the usefulness of these markers for genetic analysis of $A$. candida. Thus, we produced two populations to determine the genetic mechanisms controlling avirulence in A. candida. They were derived from crosses MiAc2-B1 × MsAc7-A1 (Cross-1) and MiAc2-B5 × MsAc7-A1 (Cross-2). Because A. candida is homothallic, these two progeny populations were expected to consist of both selfed as well as hybrid spores. The main objectives of this study were to (i) identify $\mathrm{F}_{1}$ hybrids by phenotypic screen and confirm them with RAPD markers, (ii) elucidate the genetic mechanism of avirulence of $\mathrm{F}_{2}$ populations on $B$. rapa $\mathrm{cv}$. Torch, and (iii) analyze the segregation of AFLP markers in $F_{2}$ individuals derived from two $F_{1}$ hybrids (Cr2-5 and $\mathrm{Cr} 2-7)$ of Cross-2.

\section{MATERIALS AND METHODS}

Selection of fungal isolates. The pathogenic races of $A$. candida are defined phenotypically on a set of differentials from Brassica spp. $(27,28)$. The most common races of $A$. candida in western Canada are Ac2 and Ac7. Ac2 was mainly virulent to $B$. juncea cultivars, whereas most Ac7 isolates were primarily virulent to B. rapa (28). Both Ac2 and Ac7 were homothallic and were originally sensitive to metalaxyl (18). To obtain metalaxyl-resistant isolates, sporangiospores of Ac2 and Ac7 were inoculated in the field plots that had been intensively treated with metalaxyl.

The parental isolates of $A$. candida used for genetic crosses in this investigation were collected from susceptible cultivars of $B$. juncea and $B$. rapa grown in Manitoba, Canada. Single-pustule isolates (SPI) from these collections were developed and tested for virulence to their respective susceptible hosts and for sensitivity to metalaxyl in vitro. Two metalaxyl-insensitive (Mi) isolates, MiAc2-B1 and MiAc2-B5, and metalaxyl-sensitive (Ms) isolate MsAc7-A1 were selfed for two generations on their respective susceptible hosts. Both MiAc2-B1 and MiAc2-B5 isolates belong to Ac2 of A. candida, and isolate MsAc7-A1 is Ac7. Furthermore, MiAc2-B1 showed identical background genotype to MiAc2-B5 when compared using RAPD primers (38). In addition to the differences in their avirulence or virulence to $B$. juncea $\mathrm{cv}$. Burgonde and $B$. rapa $\mathrm{cv}$. Torch and their ability to show differential metalaxyl sensitivity in vitro (Table 1), a specific representative of each race of $A$. candida had an ability to produce abundant sporangiospores and oospores on a susceptible host, and showed extensive DNA polymorphisms in RAPD analysis (38).

Host materials. Common susceptible hosts (CS) were two rapid-cycling lines representing $B$. juncea (CrGC4-1) and B. rapa (CrGC1-18). Seed of CS were provided by P. H. Williams, University of Wisconsin, Madison. Two oilseed Brassica cultivars, $B$. juncea $\mathrm{cv}$. Burgonde and B. rapa $\mathrm{cv}$. Torch, were used to determine the avirulence or virulence of parental isolates and $F_{1}$ progeny of $A$. candida. Segregation of avirulence of $\mathrm{F}_{2}$ populations was assessed on B. rapa $\mathrm{cv}$. Torch.

Crossing of fungal isolates. MiAc2-B1 and MiAc2-B5 were co-inoculated individually with MsAc7-A1 on the CS to obtain sexual recombinants. Approximately 50 plants at the beginning of the bud stage (growth stage 3.1) (12) were spray inoculated with zoospores ( 1 to $2 \times 10^{5} / \mathrm{ml}$ ) consisting of approximately equal proportions of one of the MiAc2 isolates and MsAc7. Inoculated plants were incubated in a mist chamber at $20^{\circ} \mathrm{C}$ for $48 \mathrm{~h}$ and were transferred to a growth chamber. Plants with deformed florets or racemes were moved into a greenhouse and grown under dry conditions to enhance formation and maturation of stagheads (plant hypertrophies caused by the pathogen). Stagheads containing numerous mature oospores were obtained 6 to 7 weeks after inoculation. Unless otherwise stated, oospores were germinated using the method described previously (21). Suspensions of zoospores released from oospores were filtered through Miracloth (Calbiochem Corporation, La Jolla, CA) and inoculated onto cotyledons at a low inoculum concentration ( 1 to $2 \times 10^{2}$ zoospores per $\mathrm{ml}$ ) to obtain SPI. To increase the frequency of sexual recombinants in MiAc2-B1 $\times$ MsAc7-A1 (Cross-1) or MiAc2-B5 $\times$ MsAc7-A1 (Cross-2) populations, inoculated cotyledons were sprayed with $200 \mathrm{mg}$ a.i. per milliliter of metalaxyl 2 days after inoculation to eliminate selfed progeny of the metalaxyl-sensitive parental isolate, MsAc7-A1. SPI were collected from cotyledons before the epidermis of infected host tissue ruptured. To ensure genetic purity, each SPI was subcultured individually on cotyledons of their susceptible hosts for three asexual generations (with a new SPI collected and used to initiate each generation). The SPI obtained from the third generation were increased by subculturing for two more asexual generations to obtain sufficient zoosporangia for genetic analysis. Between 10 and $12 \mathrm{~F}_{1}$ SPI were developed from each of the two putative crosses (co-inoculations), and zoosporangia collected from them were stored individually in gelatin capsules in glass screw-capped vials at $-20^{\circ} \mathrm{C}$. To obtain $\mathrm{F}_{2}$ populations, oospores from the $\mathrm{F}_{1}$ SPI were selfed on the CS and more than $50 \mathrm{~F}_{2}$ SPI were obtained and increased from each $\mathrm{F}_{1}$ SPI.

Testing specific virulence of $F_{1}$ progeny and $F_{2}$ populations. To identify $F_{1}$ hybrids, zoospores from the increased $F_{1}$ SPI were tested for their virulence to cvs. Burgonde and Torch. Hybrids from cross-fertilization between race Ac2 and Ac7 were expected to be virulent only on the CS if avirulence was dominant to virulence, or to be virulent to cvs. Burgonde and Torch as well as the $\mathrm{CS}$ if virulence was dominant to avirulence.

In our preliminary study and previous work (15), Burgonde showed variable intermediate interaction phenotypes whereas Torch was consistent when tested with different crosses of $A$. candida. As a result, Torch was selected and used as a host differential to analyze $\mathrm{F}_{2}$ populations of $A$. candida. Two seeds of Burgonde, CrGC4-1 (B. juncea CS) and Torch, CrGC1-18 (B. rapa CS) were planted in each of three cells of 12-celled multipots containing Metromix (W. R. Grace and Co., Canada Ltd., Ajax, $\mathrm{ON}$, Canada) and maintained in a growth room with day and night temperatures of 22 and $16^{\circ} \mathrm{C}$, respectively, and a 16-h photoperiod. Inoculum was prepared by germinating zoosporangia in $1 \mathrm{ml}$ of sterile, distilled water at $12^{\circ} \mathrm{C}$ for 4 to $5 \mathrm{~h}$, and the concentration was adjusted to $1 \times 10^{5}$ zoospores per ml. Six-day-old seedlings were inoculated by applying a $10-\mu$ drop of zoospore suspension on the adaxial surface of each cotyledon with an Eppendorf Repeater pipette (Brinkman Instruments Inc., Rexdale, ON, Canada). The inoculated seedlings were incubated for $24 \mathrm{~h}$ in a mist chamber and then returned to a growth chamber.

To test virulence, 12 seedlings of each cultivar were inoculated with parental isolates, $F_{1}$ progeny, and $F_{2}$ populations. All tests were carried out at least twice. The interaction phenotypes (IP) were assessed on each differential cultivar using a 0-to-9 scale 7 days after inoculation (37). Interaction phenotypes of 0 (no visible observable infection), 1, 2, and 3 (cotyledons showing no sporulation with minute to small necrotic flecks on the adaxial surface) were classified as avirulent (incompatible) phenotypes, whereas IP between 4 and 9 (cotyledons showing scattered or coalescing pustules with some to extensive sporulation on the abaxial or adaxial surface) were considered virulent (compatible).

DNA extraction. DNA was extracted from freeze-dried sporangia of parental isolates, $F_{1}$ progeny, and $104 \mathrm{~F}_{2}$ individuals from Cross- 2 using the method described by Dellaporta et al. (4). DNA was adjusted to a final concentration of $2.5 \mathrm{ng} / \mu \mathrm{l}$ and $25 \mathrm{ng} / \mu \mathrm{l}$ for RAPD and AFLP analysis, respectively.

Confirming $F_{1}$ hybrids with RAPD markers. To identify RAPD markers specific to the parents, we screened 300 commercially available RAPD decamer primers obtained from the University of British Columbia (UBC), BC, Canada. PCR reactions $(20 \mu \mathrm{l})$ contained $4 \mu \mathrm{l}$ of DNA $(2.5 \mathrm{ng} / \mu \mathrm{l}), 2 \mu \mathrm{l}$ of $10 \mathrm{mM}$ dNTP mix (Amersham Pharmacia Biotech, Piscataway, NJ), $1.5 \mu \mathrm{l}$ of $25 \mathrm{mM} \mathrm{MgCl}_{2}$ (Promega Corp., Madison, WI), $2 \mu \mathrm{l}$ of $10 \times$ PCR buffer (Promega), 1 unit of Taq DNA polymerase (Promega), $4 \mu \mathrm{l}$ 
of $2 \mu \mathrm{M}$ primer mixture, and $6.4 \mu \mathrm{l}$ of sterile water (36). All reactions were carried out in a PTC-200 thermocycler (MJ Research Inc., Waltham, MA). Each amplification cycle consisted of denaturation at $94^{\circ} \mathrm{C}$ for $1 \mathrm{~min}$, annealing step at $37^{\circ} \mathrm{C}$ for $1 \mathrm{~min}$, and extension step at $72^{\circ} \mathrm{C}$ for $2 \mathrm{~min}$. After 45 cycles, an extra extension step was performed for $10 \mathrm{~min}$ at $72^{\circ} \mathrm{C}$. The PCR products were separated by electrophoresis in $2 \%$ agarose gels with $0.5 \times$ TAE $(0.02 \mathrm{M}$ Tris, $0.0095 \mathrm{M}$ glacial acetic acid, and 0.005 M EDTA, pH 8.0) buffer. A low-DNA mass ladder or a 1-kb ladder (Gibco, BRL, Burlington, ON, Canada) was used as a size marker. Gels were run at $3 \mathrm{~V} / \mathrm{cm}$ and DNA bands were photographed. RAPD markers were named by UBC primer followed by the molecular size (in kilobase pairs) of each band. For example, UBC717 (2.8) is the 2.8-kb polymorphic band generated by primer UBC717.

Candidate polymorphisms were checked for reproducibility in several preparations of miniprep DNAs from the two parents. To confirm $\mathrm{F}_{1}$ hybrids, the $10 \mathrm{~F}_{1}$ progeny derived from Cross-2 $(\mathrm{MiAc} 2-\mathrm{B} 5 \times$ MsAc7-A1) were tested with RAPD primers UBC415, UBC733, and UBC792. For example, UBC415 detected two markers $(2.0$ and $1.0 \mathrm{~kb})$ specific to the MiAc2-B5 parent and one marker $(0.8 \mathrm{~kb})$ specific to the MsAc7-A1 parent.

Segregation of AFLP markers in $\mathbf{F}_{2}$ populations. Bulked segregant analysis (BSA) was performed to identify potential AFLP markers (23). DNA from five avirulent and five virulent $F_{2}$ progeny were pooled to constitute avirulent and virulent bulks. AFLP analysis was conducted using AFLP Analysis System I, AFLP Start Primer Kit (Gibco-BRL, Life Technologies, Inc., Gaithersburg, MD), as described by Vos et al. (33). Briefly, $10 \mu \mathrm{l}$ of genomic DNA $(25 \mathrm{ng} / \mu \mathrm{l})$ of each parent and $\mathrm{F}_{2}$ progeny was digested in a reaction volume of $25 \mu \mathrm{l}$ with $2.5 \mathrm{U}$ each of EcoRI and MseI restriction enzyme, and ligated to EcoRI and MseI adapters. The digested DNA, together with 2 pmol of EcoRI adapter and $20 \mathrm{pmol}$ of $\mathrm{MseI}$ adapter, were ligated with 1 unit of $\mathrm{T} 4$ ligase. The ligation mix was incubated at $20^{\circ} \mathrm{C}$ for $2 \mathrm{~h}$ and then the enzyme was inactivated at $70^{\circ} \mathrm{C}$ for $15 \mathrm{~min}$. The ligated product was diluted 10-fold in TE (10 mM Tris-HC, pH 8.0, $0.1 \mathrm{mM}$ EDTA). Preamplification, primer labeling, and selective amplification was performed according to Vos et al. (33). All reactions were carried out in a PTC-200 thermocycler (MJ Research Inc.). Each sample ( $3 \mu \mathrm{l})$ was loaded onto a $5 \%$ denaturing polyacrylamide-sequencing gel and run in $1 \times$ TBE $(0.089 \mathrm{M}$ Tris, $0.089 \mathrm{M}$ boric acid, and $0.002 \mathrm{M}$ EDTA) buffer at $90 \mathrm{~W}$ for $3 \mathrm{~h}$. A 500-bp ladder (Gibco BRL) was used as a size marker. The gel was dried at $80^{\circ} \mathrm{C}$ for $2 \mathrm{~h}$ and exposed on X-ray film (Kodak Co., Toronto) at $-70^{\circ} \mathrm{C}$ for 2 to 3 days, depending on the signal intensity. The AFLP bands were scored by visual inspection. AFLP loci were named based on the combination of selective nucleotides in each primer (EcoRI and $M s e \mathrm{I}$ ) and the size of the band. For example, AGG/CAT 1 refers to the first polymorphic band (bands numbered from high to low molecular weight) amplified with the primer combination EcoRI-AGG and MseI-CAT.

To analyze segregation of AFLP markers in A. candida, 20 AFLP primer combinations with two and three selective nucleotides at the $3^{\prime}$ end were tested for polymorphism in the two parents (MiAc2-B5 and MsAc7-A1) and two bulks. Among those, the EcoRI-AA and MseI-CT primer combination (hereafter referred to as E-AA/M-CT) was selected and used to analyze genomic DNA of the $104 \mathrm{~F}_{2}$ individuals from $\mathrm{F}_{1} \mathrm{~s}(\mathrm{Cr} 2-5$ and $\mathrm{Cr} 2-$ 7) of Cross-2 (Table 1).

Data analysis. Chi-square values were calculated to compare the observed ratios of avirulent versus virulent infection phenotypes against Mendelian ratios of 3:1 that would be expected if the avirulence were controlled by a single dominant gene. Chi-square

TABLE 1. Identification of hybrids and selfed progeny from $\mathrm{F}_{1}$ crosses between isolates of Albugo candida by virulence and metalaxyl insensitivity testing

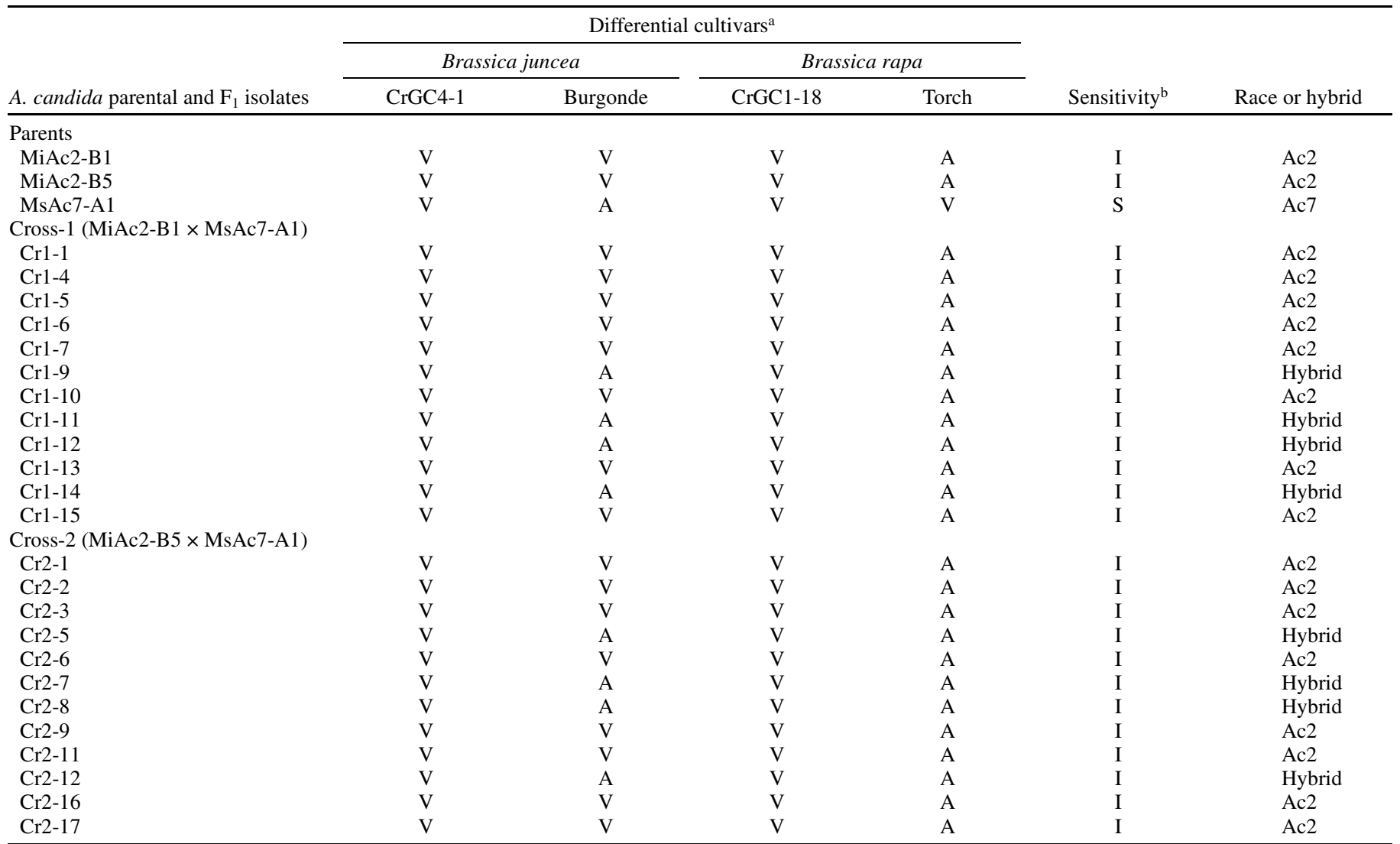

${ }^{\mathrm{a}} \mathrm{CrGC4}-1$ and $\mathrm{CrGC} 1-18$ are common susceptible hosts to races Ac2 and Ac7. A = avirulent if interaction phenotype (IP) 0-3; V = virulent if IP 4. Values represent the mean of ratings on 24 to 30 seedlings.

${ }^{\mathrm{b}}$ Sensitivity to metalaxyl; I = Insensitive to metalaxyl; S = Sensitive. 
values also were used to compare the segregation ratios of AFLP markers, and $P<0.05$ was selected as the level for rejecting the null hypothesis.

\section{RESULTS}

Parent isolates. Hybrid progeny were distinguished from those originating by self fertilization because all the SPI derived from selfed parental isolates MiAc2-B1 and MiAc2-B5 were insensitive to metalaxyl, virulent to Burgonde, and avirulent to Torch (Table 1). Lack of segregation in the selfed progeny indicated that the parent isolates were homozygous for alleles controlling virulence and metalaxyl insensitivity. All SPI obtained from selfing the MsAc7-A1 parent were sensitive to metalaxyl, avirulent to Burgonde, and virulent to Torch, indicating that this isolate was homozygous for these traits. Therefore, both homozygous isolates MiAc2-B1 and MiAc2-B5 were used for crossing with MsAc7-A1.

Specific virulence of $\mathbf{F}_{\mathbf{1}}$ hybrids. Of $12 \mathrm{~F}_{1}$ progeny derived from Cross-1, four (Cr1-9, Cr1-11, Cr1-12, and Cr1-14) were either avirulent to both differential cultivars or showed reduced virulence on Burgonde (Table 1). The remaining eight $\mathrm{F}_{1}$ progeny from Cross-1 (Cr1-1, Cr1-4, Cr1-5, Cr1-6, Cr1-7, Cr1-10, Cr1-13, and $\mathrm{Cr} 1-15)$ did not differ in virulence from the parental isolate MiAc2-B1, suggesting that they probably resulted from self fertilization (Table 1). Similarly, only four $F_{1}$ progeny in Cross- 2 (Cr2-5, Cr2-7, Cr2-8, and Cr2-12) were avirulent to both Torch and Burgonde. No isolates virulent to both of these cultivars were recovered from either of the two crosses, suggesting that avirulence was dominant.

Segregation of avirulence or virulence in the $F_{2}$ populations. To test the hypothesis that avirulence was dominant and controlled by a single gene, three $\mathrm{F}_{2}$ populations derived from Cross- 1 (Cr19, Cr1-11, and Cr1-14) and three $F_{2}$ populations from selfing of $F_{1}$ progeny of Cross-2 (Cr2-5, $\mathrm{Cr} 2-7$, and $\mathrm{Cr} 2-8)$ were tested for virulence on Torch. The segregation for avirulence fit a ratio of 3:1 (avirulent/virulent) on Torch for all six $F_{2}$ populations, indicating that avirulence in Ac2 appears to be governed by a single, dominant gene (Table 2).

Confirmation of $\mathbf{F}_{\mathbf{1}}$ hybrids with RAPD markers. Of the 300 RAPD primers tested, only $27(9 \%)$ showed polymorphism between the two parental isolates. The molecular size of the markers ranged from 0.5 to $2.6 \mathrm{~kb}$. To confirm the putative $F_{1}$ hybrids, identified by phenotypic screening, a marker that produced a band in MiAc2-B5, but not in MsAc7-A1, always was tested with a second marker, which produced a band in MsAc7-A1 but not in MiAc2-B5. Primers used for this purpose included UBC415, which amplified two bands (Fig. 1), one specific to MiAc2-B5 $(2.0 \mathrm{~kb})$ and another specific to MsAc7-A1 (0.8 kb). Other primers used that were specific to MiAc2-B5 were UBC733 $(1.6 \mathrm{~kb})$ and $\mathrm{UBC} 792(1.6 \mathrm{~kb})$. The $10 \mathrm{~F}_{1}$ progeny, derived only from Cross-2, were analyzed and $4 \mathrm{~F}_{1}$ progeny $(\mathrm{Cr} 2-5, \mathrm{Cr} 2-7, \mathrm{Cr} 2-8$, and $\mathrm{Cr} 2$-12) exhibited bands from both parents, confirming the phenotypic data that these isolates were hybrids (Fig. 1).
Segregation of AFLP markers in $\mathbf{F}_{2}$ populations. Segregation of an AFLP marker combination (E-AA/M-CT) was determined in the $104 \mathrm{~F}_{2}$ individuals from Cross-2 (Fig. 2). Four AFLP markers (E-AA/M-CT-2, E-AA/M-CT-4, E-AA/M-CT-5, and E-AA/M-CT6) were polymorphic and segregated in regular Mendelian fashion. The segregation ratios fit 3:1 for presence or absence of the marker in combination or individually (Table 3).

\section{DISCUSSION}

This research demonstrated that $A$. candida, a homothallic oomycete, could form hybrids between genetically distinct isolates via co-culture. This phenomenon has been described in other homothallic oomycetes $(31,35)$ after its first demonstration in $P y$ thium ultimum (11) and it is important to report that this also can happen in A. candida.

Analysis of $\mathrm{F}_{2}$ populations showed that avirulence against Torch segregated in 3:1 ratios, indicating that a single locus apparently controlled avirulence against Torch. This was consistently observed in $\mathrm{F}_{2}$ populations derived from all six $\mathrm{F}_{1}$ hybrids from two crosses. Thus our hypothesis is that there is a single dominant gene in race Ac2 to confer specific avirulence to Torch. We are designating this first putative avirulence gene AvrAcl. Segregation of avirulence also has been reported in Phytophthora infestans $(29,30)$, Peronospora parasitica (10), and Phytophthora sojae $(31,34,35)$. In another oomycete, Bremia lactucae, which causes lettuce downy mildew, a number of single dominant avirulence genes have been identified, which segregated in regular Mendelian fashion (3,14,22,24).

Segregation data indicated that host resistance to race Ac2 of $A$. candida in Brassica rapa cv. Torch is controlled by two dominant

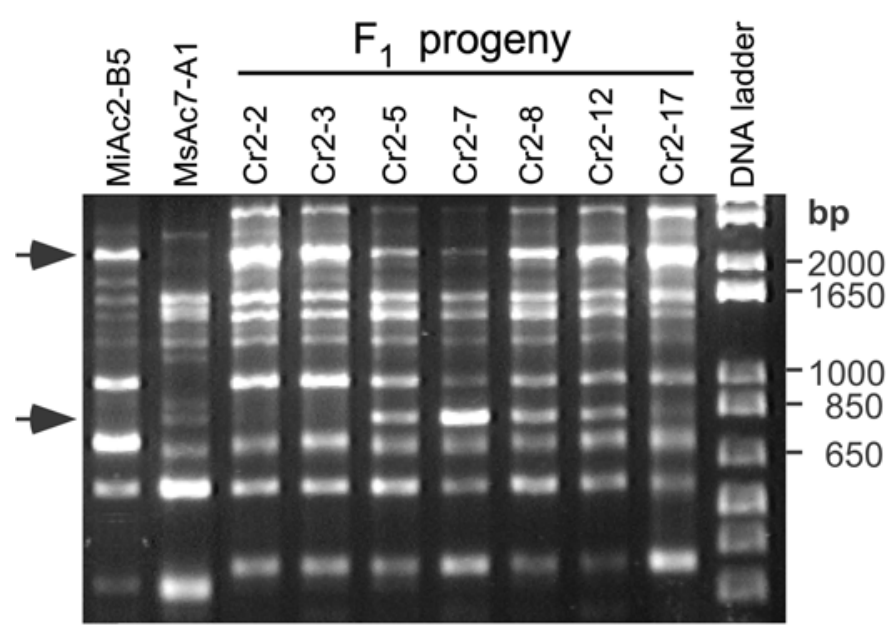

Fig. 1. Detection of $\mathrm{F}_{1}$ hybrids from the cross MiAc2-B5 $\times$ MsAc7-A1 of Albugo candida by random amplified polymorphic DNA (RAPD) markers. Primer UBC415 amplified two bands: upper arrow specific to MiAc2-B5, and lower arrow specific to MsAc7-A1. Four F 1 hybrids, Cr2-5, Cr2-7, Cr2-8 and $\mathrm{Cr} 2-12$, display both parent-specific RAPD bands.

TABLE 2. Segregation of avirulence among $\mathrm{F}_{2}$ populations of Albugo candida on Brassica rapa cv. Torch

\begin{tabular}{|c|c|c|c|c|}
\hline \multirow[b]{2}{*}{ A. candida crosses and $\mathrm{F}_{2}$ populations } & \multicolumn{4}{|c|}{ B. rapa cv. Torch } \\
\hline & $\mathrm{F}_{2}$ ratio $(\mathrm{A}: \mathrm{V})^{\mathrm{a}}$ & Expected ratio & $\chi^{2}$ & Probability \\
\hline \multicolumn{5}{|l|}{ Cross-1 (MiAc2-B1 × MsAc7-A1) } \\
\hline Cr1-9 & $44: 9$ & $3: 1$ & 1.87 & $0.1-0.5$ \\
\hline Cr1-11 & $40: 11$ & $3: 1$ & 0.32 & $0.5-0.9$ \\
\hline $\mathrm{Cr} 1-14$ & $54: 12$ & $3: 1$ & 1.63 & $0.1-0.5$ \\
\hline \multicolumn{5}{|l|}{ Cross-2 (MiAc2-B5 × MsAc7-A1) } \\
\hline $\mathrm{Cr} 2-5$ & $40: 8$ & $3: 1$ & 1.77 & $0.1-0.5$ \\
\hline $\mathrm{Cr} 2-7$ & $46: 10$ & $3: 1$ & 1.52 & $0.1-0.5$ \\
\hline $\mathrm{Cr} 2-8$ & $44: 9$ & $3: 1$ & 1.81 & $0.1-0.5$ \\
\hline
\end{tabular}

a $\mathrm{A}=$ avirulent and $\mathrm{V}=$ virulent. 
genes (15). It is not known whether these two genes are the same major resistance gene or closely linked genes. It is possible that both genes in Torch were involved in the specificity to recognize a single avirulence gene in $A$. candida, identified in crosses of race Ac2 and Ac7 (15). Genetic control of avirulence by a single gene is common in many plant-pathogen interactions (6). Generally, avirulence genes determine specificity to individual resistance genes and support the gene-for-gene hypothesis (9). Previous suggestions of a gene-for-gene relationship between Brassica spp. and $A$. candida $(15,19,20)$ are supported by the present data.

Hybridization between pathogenic races of $A$. candida probably occurs frequently in nature, provided suitable host genotypes are present (28). Outcrossing in the field would be expected to recombine genetic variability in A. candida populations. In another oomycete, Pythium ultimum, sexual populations were observed to be more genetically diverse than asexual populations, and a higher level of genetic variation corresponded to greater diversity in specific virulence (11). Our study has shown that hybridization between two races of $A$. candida can occur under greenhouse conditions. The frequency of recombinant isolates was $33 \%$ for both Cross-1 and Cross-2 after elimination of the metalaxylsensitive parental selfs. Thus, assuming a similar frequency of selfing in the metalaxyl-insensitive parent as occurred in the metalaxyl-sensitive parent, $\approx 20 \%$ of the $\mathrm{F}_{1}$ isolates were hybrids and $\approx 80 \%$ were parental types resulting from selfing. A very high frequency mitotic gene conversion $\left(\approx 5 \times 10^{-5}\right.$ per locus per nucleus per generation) also was observed in a cross P6497 $\times$ P7064 of $P$. sojae (2). It was further suggested that this mechanism would result in a large reservoir of diversity within an individual thallus that could facilitate a strain to adapt to new environmental challenges (2).
We hypothesized that $F_{1}$ hybrids could be obtained directly by treating with metalaxyl to eliminate of MsAc7-A1, then inoculating onto B. rapa cv. Torch to eliminate the MiAc2-B1 or MiAc2B-5 isolates. Anything surviving should be an $\mathrm{F}_{1}$ hybrid, assuming that both virulence and metalaxyl resistance are at least semidominant. In the present study, 4 of 12 progeny (33\%) were $\mathrm{F}_{1}$ hybrids from both Cross- 1 and Cross- 2 . Approximately $67 \%$ of $F_{1}$ progeny of our crosses were isolates of Ac2, but no Ac7 was detected. The data presented here, together with those of Tyler et al. (31) and Whisson et al. (34,35), suggested that this obligate fungus, A. candida, is capable of producing hybrids in vivo when different races are co-inoculated together. Compared with heterothallic fungi such as $P$. infestans and $P$. parasitica, homothallic isolates of $A$. candida also can form oospores readily by selfing and individual strains should be homozygous, as a result of repeated selfing. It is advantageous to use genetic markers to identify $F_{1}$ hybrids among the progeny in this species. Here, we used RAPD markers to confirm the phenotypic screen used to identify $\mathrm{F}_{1}$ hybrids in $A$. candida. RAPD data showed that all $\mathrm{F}_{1}$ hybrids contained alleles from both parents. Several studies have been conducted to investigate genetic analysis of $P$. sojae to confirm the $F_{1}$-hybrid nature of the progeny by different genetic markers $(1,31,35)$. Bhat and Schmitthenner (1) used drug resistance markers to identify $F_{1}$ hybrids from crosses of different races of $P$. sojae. Whisson et al. (35) reported evidence of single dominant avirulent genes against Rpsla, Rps $3 a$, Rps5, and Rps6, using a cross in which an $F_{1}$ hybrid was identified by RAPD markers specific for each parent. Tyler et al. (31) identified $F_{1}$ hybrids from crosses of three isolates (P6497, P7064, and P7076) by RAPD markers. In addition, RFLP markers have been used to verify the hybrid nature of progeny from crosses between $P$.

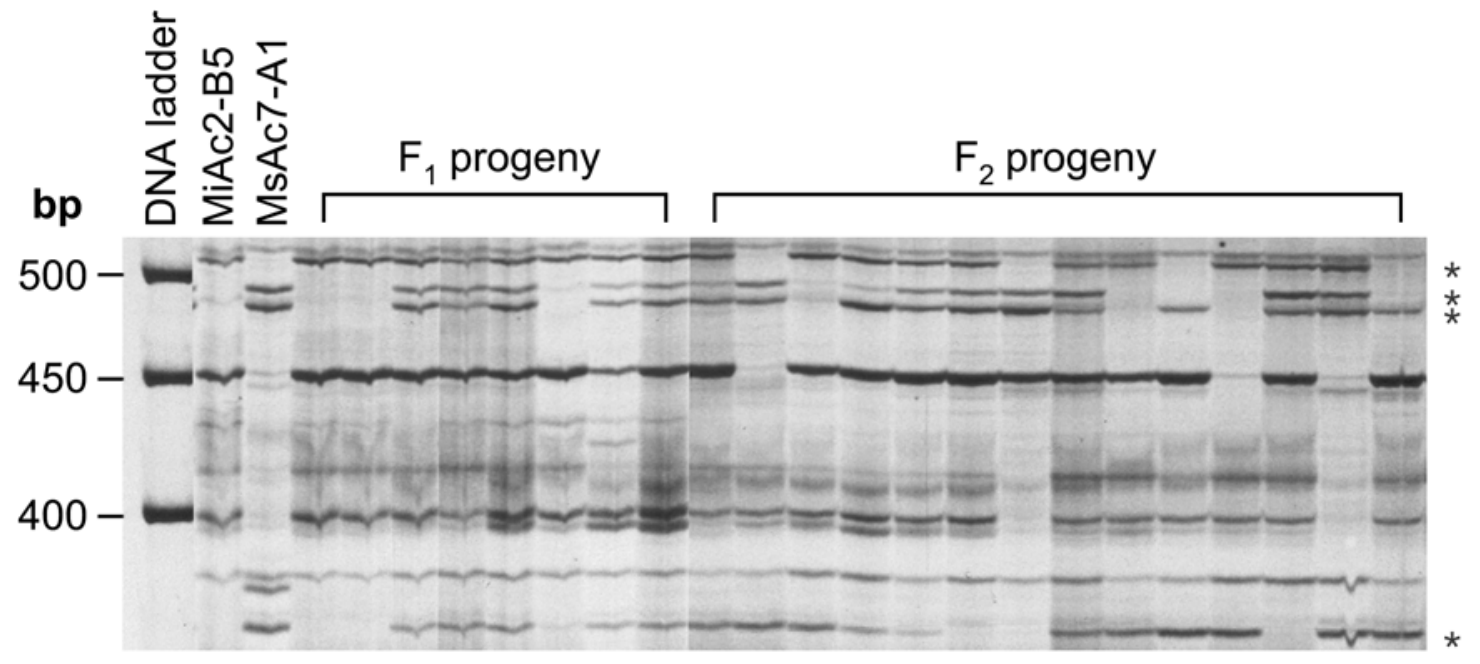

Fig. 2. Segregation of amplified fragment length polymorphisms (AFLP) markers among $\mathrm{F}_{2}$ progeny of Albugo candida (MiAc2-B5 $\times$ MsAc7-A1). Primer combination E-AA/M-CT detects polymorphism (stars) in MiAc2-B5 and MsAc7-A1. The $\mathrm{F}_{2}$ progeny display segregation of these AFLP markers.

TABLE 3. Segregation of amplified fragment length polymorphism (AFLP) markers in the $\mathrm{F}_{2}$ populations of Albugo candida

\begin{tabular}{|c|c|c|c|c|c|c|c|c|c|}
\hline \multirow[b]{3}{*}{ AFLP markers $^{\mathrm{a}}$} & \multicolumn{8}{|c|}{ Segregation of $\mathrm{F}_{2}$ populations ${ }^{\mathrm{b}}$} & \multirow[b]{3}{*}{ Probability } \\
\hline & \multicolumn{4}{|c|}{ Cross 2-5 } & \multicolumn{4}{|c|}{ Cross 2-7 } & \\
\hline & + & - & Ratio +:- & $\chi^{2}$ & + & - & Ratio +:- & $\chi^{2}$ & \\
\hline E-AA/M-CT4 & 37 & 11 & $3: 1$ & 0.11 & 40 & 16 & $3: 1$ & 0.38 & $\mathrm{~ns}$ \\
\hline E-AA/M-CT5 & 39 & 9 & $3: 1$ & 1.00 & 48 & 18 & $3: 1$ & 3.43 & $\mathrm{~ns}$ \\
\hline E-AA/M-CT6 & 36 & 12 & $3: 1$ & 0.00 & 42 & 14 & $3: 1$ & 0.00 & ns \\
\hline
\end{tabular}

${ }^{a}$ Each marker band was scored against 104 progeny pooled from two $\mathrm{F}_{1} \mathrm{~s}$ of Cross-2 (48 progeny from $\mathrm{Cr} 2-5$ and 56 progeny from $\mathrm{Cr} 2-7$ ).

${ }^{\mathrm{b}}$ Band absent $=-$, band present $=+$. Goodness of fit $\left(\chi^{2}\right)$ of the ratios of the different markers in $\mathrm{F}_{2}$ progeny expected for single dominant markers $(3: 1)$; $\chi^{2}$ values in excess of $95 \%$ significance levels (3.84 for two classes) are marked with an asterisk.

${ }^{\mathrm{c}}$ Combined probability; $\mathrm{ns}=$ not significant. 
parasitica isolates (10). In the present study, we analyzed $10 \mathrm{~F}_{1}$ progeny from Cross-2 to confirm putative hybrids of $A$. candida with RAPD markers.

The polymorphic nature of RAPDs and segregation ratios of AFLP markers in the $F_{2}$ individuals from two populations analyzed in this study indicated that the three parental isolates were homozygous for the markers tested. Tyler et al. (31) and Whisson et al. (35) also observed no evidence for heterozygosity in their crosses of $P$. sojae. However, these observations do not rule out that $F_{1}$ hybrids may undergo frequent gene conversion resulting in some genetic markers becoming homozygous during vegetative maintenance. Changes from heterozygosity to homozygosity occur at high frequency during vegetative growth of certain $P$. sojae hybrids (2). It was further suggested that high-frequency mitotic gene conversion rapidly converts heterozygous loci to homozygosity, resulting in heterokaryons containing highly diverse populations of diploid nuclei (2).

PCR-based technology has been successfully applied to detect genetic variation and linkage groups of many plant-pathogenic fungi, including obligate fungal pathogens of agriculturally important crops, even though the difficulty of collecting tissue imposes constraints on the number of individuals sampled. Both RAPD and AFLP markers have been used recently in a wide range of studies, including mapping of disease resistance genes in hosts and avirulence genes in plant pathogens. Although different molecular marker systems have been used successfully to investigate genetic analysis in other oomycetes $(10,14,31,34,35)$, no attempt has been made to examine segregation of molecular markers in A. candida until now. In this study, RAPD markers showed an unusually high frequency of biased segregation in two $\mathrm{F}_{2}$ populations derived from different $\mathrm{F}_{1} \mathrm{~s}$ from the same cross (data not shown). Thus, the main goal of this research was to find suitable polymorphic AFLP markers for genetic analysis of $A$. candida. We selected an AFLP primer combination, EcoRIAA/MseI-CT, which was polymorphic between two parents and bulks, and was used to analyze the two $F_{2}$ populations from $F_{1}$ hybrids of Cross-2. The segregation of four AFLP markers analyzed in this study showed normal segregation ratios. Several groups working on oomycetes also found that molecular markers segregated in a Mendelian fashion $(14,30,34,35)$. For segregation of genetic markers, $\mathrm{F}_{2}$ individuals from two $\mathrm{F}_{1}$ hybrids $(\mathrm{Cr} 2-5$ and $\mathrm{Cr} 2-7$ ) from Cross-2 were tested. Analysis of other $\mathrm{F}_{2}$ individuals from $F_{1}$ hybrids from Cross- 1 and Cross- 2 with AFLP markers will be required to demonstrate whether there is normal segregation or biased segregation in those populations. We also are in the process of developing $\mathrm{F}_{3}$ populations by selecting the avirulent $\mathrm{F}_{2}$ progeny and applying AFLP markers to construct a genetic linkage map of $A$. candida based on $\mathrm{F}_{3}$ populations. This would help us to place AFLP markers on the linkage map and to identify closely linked genetic markers to the putative avirulence gene AvrAcl, identified in this study, and other avirulence loci.

\section{ACKNOWLEDGMENTS}

The Natural Sciences and Engineering Research Council of Canada, the Canola Council of Canada, Agrium Inc., and the Matching Investment Initiative of Agriculture and Agri-Food Canada supported this research. We thank S. Goodwin for valuable comments and suggestions, and G. Ardiel and P. Parks for technical assistance.

\section{LITERATURE CITED}

1. Bhat, R. G., and Schmitthenner, A. F. 1993. Genetic crosses between physiologic races of Phytophthora sojae. Mycol. Res. 97:865-870.

2. Chamnanpunt, J., Shan, W.-X., and Tyler, B. M. 2001. High frequency mitotic gene conversion in genetic hybrids of the oomycete Phytophthora sojae. Proc. Natl. Acad. Sci. USA 98:14530-14535.

3. Crute, I. R., and Johnson, A. G. 1976. The genetic relationship between races of Bremia lactucae and cultivars of Lactuca sativa. Ann. Appl.
Biol. 83:125-137.

4. Dellaporta, S. L., Wood, J., and Hicks, J. B. 1983. A plant DNA minipreparation: Version II. Plant Mol. Biol. Rep. 1:19-21.

5. Delwiche, P. A., and Williams, P. H. 1977. Genetic studies in Brassica nigra (L.) Koch. Cruciferae Newsl. 2:39.

6. De Wit, P. J. G. M. 1997. Pathogen avirulence and plant resistance: A key role for recognition. Trends Plant Sci. 2:425-428.

7. Fan, Z., Rimmer, S. R., and Stefansson, B. R. 1983. Inheritance of resistance to Albugo candida in rape Brassica napus L. Can. J. Genet. Cytol. 25:420-424.

8. Ferreira, M. E., Williams, P. H., and Osborn, T. C. 1995. Mapping of a locus controlling resistance to Albugo candida in Brassica napus using molecular markers. Phytopathology 85:218-220.

9. Flor, H. H. 1971. Current status of the gene-for-gene concept. Annu. Rev. Phytopathol. 9:275-296.

10. Förster, H., and Coffey, M. D. 1990. Mating behavior of Phytophthora parasitica-evidence for sexual recombination in oospores using DNA restriction fragment length polymorphism as genetical markers. Exp. Mycol. 14:351-359.

11. Francis, D. M., and St. Clair, D. A. 1993. Outcrossing in the homothallic oomycete, Pythium ultimum, detected with molecular markers. Curr. Genet. 24:100-106.

12. Harper, F. R., and Berkenkamp, B. 1975. Revised growth stage key for Brassica campestris and B. napus. Can. J. Plant Sci. 55:657-658.

13. Hill, C. B., Crute, I. R., Sheriff, C., and Williams, P. H. 1988. Specificity of Albugo candida and Peronospora parasitica pathotypes towards rapid-cycling crucifers. Cruciferae Newsl. 13:112-113.

14. Hulbert, S. H., Illott, T. W., Legg, E. J., Lincoln, S. E., Lander, E. S., and Michelmore, R. W. 1988. Genetic analysis of the fungus, Bremia lactucae, using restriction fragment length polymorphisms. Genetics 120:947-958.

15. Kalavacharla, V. S., Wu, C. R., and Rimmer, S. R. 1999. Genetic analysis for resistance to Albugo candida in Brassica differentials. Paper 46 in: Proc. 10th Int. Rapeseed Congr., GCIRC, Canberra, Australia.

16. Kole, C., Teutonico, R., Mengistu, A., Williams, P. H., and Osborn, T. C. 1996. Molecular mapping of a locus controlling resistance to Albugo candida in Brassica rapa. Phytopathology 86:367-369.

17. Kole, C., Williams, P. H., Rimmer, S. R., and Osborn, T. C. 2002. Linkage mapping of genes controlling resistance to white rust (Albugo candida) in Brassica rapa (syn. campestris) and comparative mapping to Brassica napus and Arabidopsis thaliana. Genome 45:22-27.

18. Liu, Q. 1992. A methodology for genetic analysis with Albugo candida. Ph.D. thesis. Department of Plant Science, The University of Manitoba, Winnipeg, Canada.

19. Liu, J. Q., Parks, P., and Rimmer, S. R. 1996. Development of monogenic lines for resistance to Albugo candida from a Canadian Brassica napus cultivar. Phytopathology 86:1000-1004.

20. Liu, Q., and Rimmer, S. R. 1992. Inheritance of resistance in Brassica napus to an Ethiopian isolate of Albugo candida from Brassica carinata. Can. J. Plant Pathol. 14:116-120.

21. Liu, Q., and Rimmer, S. R. 1993. Production and germination of oospores of Albugo candida. Can. J. Plant Pathol. 15:265-271.

22. Michelmore, R. W., Norwood, J. M., Ingram, D. S., Crute, I. R., and Nicholson, P. 1984. The inheritance of virulence in Bremia lactucae to match resistance factors 3, 4, 5, 6, 7, 8, 9, 10, and 11 in lettuce (Lactuca sativa). Plant Pathol. 33:301-315.

23. Michelmore, R. W., Paran, I., and Kesseli, R. V. 1991. Identification of markers linked to disease resistance genes by bulked segregant analysis: A rapid method to detect markers in specific genomic regions by using segregating populations. Proc. Natl. Acad. Sci. USA 88:9828-9832.

24. Norwood, J. M., Michelmore, R. W., Crute, I. R., and Ingram, D. S. 1983. The inheritance of specific virulence in Bremia lactucae (downy mildew) to match specific resistance factors 1, 2, 4, 6 and 11 in Lactuca sativa (lettuce). Plant Pathol. 32:177-186.

25. Petrie, G. A. 1988. Races of Albugo candida (white rust and stag head) on cultivated cruciferae in Saskatchewan. Can. J. Plant Pathol. 10:142150 .

26. Pidskalny, R. S., and Rimmer, S. R. 1985. Virulence of Albugo candida from turnip rape (Brassica campestris) and mustard (Brassica juncea) on various crucifers. Can. J. Plant Pathol. 7:283-286.

27. Pound, G. S., and Williams, P. H. 1963. Biological races of Albugo candida. Phytopathology 53:1146-1149.

28. Rimmer, S. R., Mathur, S., and Wu, C. R. 2000. Virulence of isolates of Albugo candida from western Canada to Brassica species. Can. J. Plant Pathol. 22:229-235.

29. Shaw, D. S. 1991. Genetics. Pages 131-170 in: Phytophthora infestans. Advances in Plant Pathology. Vol. 7. D. S. Ingram and P. H. Williams, eds. Academic Press, New York.

30. Spielman, L. J., Sweigard, J. A., Shattock, R. C., and Fry, W. E. 1990. The genetics of Phytophthora infestans: Segregation of allozyme mark- 
ers in $\mathrm{F}_{2}$ and backcross progeny and the inheritance of virulence against potato resistance gene R2 and R4 in $\mathrm{F}_{1}$ progeny. Exp. Mycol. 14:57-69.

31. Tyler, B. M., Förster, H., and Coffey, M. D. 1995. Inheritance of avirulence factors and restriction fragment length polymorphism markers in outcrosses of the oomycete Phytophthora sojae. Mol. Plant-Microbe Interact. 8:515-523.

32. Verma, U., and Bhowmik, P. T. 1989. Inheritance of resistance to a Brassica juncea pathotype of Albugo candida in B. napus. Can. J. Plant Pathol. 11:443-444.

33. Vos, P., Hogers, R., Bleeker, M., Reijans, M., van der Lee, T., Hornes, M., Frijters, A., Pot, J., Peleman, J., Kuiper, M., and Zabeau, M. 1995. AFLP: A new technique for DNA fingerprinting. Nucleic Acids Res. 23:4407-4414

34. Whisson, S. C., Drenth, A., Maclean, D. J., and Irwin, J. A. G. 1994. Evidence for outcrossing in Phytophthora sojae and linkage of a DNA marker to avirulence genes. Curr. Genet. 27:77-82.

35. Whisson, S. C., Drenth, A., Maclean, D. J., and Irwin, J. A. G. 1995. Phytophthora sojae avirulence genes, RAPD and RFLP markers used to construct a detailed genetic linkage map. Mol. Plant-Microbe Interact. 8:988-995.

36. Williams, J. G. K., Kubelik, A. R., Lavak, K. J., Rafalski, J. A., and Tingey, S. V. 1990. DNA polymorphisms amplified by arbitrary primers are useful as genetic markers. Nucleic Acids Res. 18:6531-6535.

37. Williams, P. H. 1985. Crucifer Genetics Cooperative (CRGC) Resource Book. Department of Plant Pathology, University of Wisconsin, Madison.

38. Wu, C. R., Mathur, S., and Rimmer, S. R. 1995. Differentiation of races and isolates of Albugo candida by random amplification of polymorphic DNA. Pages 655-657 in: Proc. 9th Int. Rapeseed Congr., GCIRC, Cambridge, UK 